\title{
Dynamin 3 suppresses growth and induces apoptosis of hepatocellular carcinoma cells by activating inducible nitric oxide synthase production
}

\author{
CHAO GU* ${ }^{*}$ JUNLIANG YAO* and PEILONG SUN \\ Department of General Surgery, Jinshan Hospital, Fudan University, Shanghai 201508, P.R. China
}

Received September 5, 2015; Accepted February 23, 2017

DOI: $10.3892 / \mathrm{ol} .2017 .6057$

\begin{abstract}
Dynamin 3 (DNM3) is candidate tumor suppressor against hepatocellular carcinoma (HCC). Downregulation of DNM3 is more frequently identified in HCC tissues than in normal liver tissues. However, the mechanism underlying DNM3-mediated inhibition of HCC remains unclear. The present study demonstrated that DNM3 expression was decreased in human HCC tissues and cell lines. The downregulation of DNM3 promoted cell proliferation by increasing cell cycle-associated proteins, including cyclin D1, cyclin-dependent kinase (CDK) 2 and CDK4. The upregulation of DNM3 induced HCC cell apoptosis and inhibited tumor growth. The present study also revealed that overexpression of DNM3 induced nitric oxide (NO) production and intracellular reactive oxygen species (ROS) accumulation. DNM3 overexpression also increased the protein expression level of inducible nitric oxide synthase (iNOS) in HCC cells and subcutaneous HCC tumor xenografts. The inhibition of iNOS by L-canavanine attenuated the DNM3-induced ROS accumulation and apoptotic cell death. In conclusion, the results indicate that DNM3 overexpression may induce apoptosis and inhibit tumor growth of HCC by activating iNOS production and the subsequent NO-ROS signaling pathways.
\end{abstract}

\section{Introduction}

Liver cancer is the second leading cause of cancer-associated mortality, and hepatocellular carcinoma (HCC) accounts for $\geq 90 \%$ of liver cancer cases (1). To date, liver resection and liver transplantation remain the most effective treatments for patients with HCC (2). However, the majority of patients do

Correspondence to: Professor Peilong Sun, Department of General Surgery, Jinshan Hospital, Fudan University, 1508 Longhang Road, Jinshan, Shanghai 201508, P.R. China

E-mail: sunpeilongsh@sina.com

\section{${ }^{*}$ Contributed equally}

Key words: dynamin 3, hepatocellular carcinoma, apoptosis, inducible nitric oxide synthase, reactive oxygen species not have the opportunity for surgery due to late diagnosis and tumor progression (3). Despite the emergence of novel therapies for HCC, the prognosis of patients with HCC remains poor $(3,4)$. The carcinogenesis of HCC is complex and involves numerous biological processes (5). The development of novel interventions for HCC requires improved understanding of the disease (6). Therefore, further research to reveal the mechanisms underlying HCC carcinogenesis is urgently required in order to improve the management of this type of cancer.

Dynamins (DNMs) are a family of guanylate triphosphatases (GTPases) that participate in vesicle budding and membrane severing via the hydrolysis of GTPs (7). DNM3 is a member of the DNM family that is essential for endocytosis and possesses mechanochemical properties that are important for actin-membrane processes (8). The multiple domains of DNMs facilitate their interaction with numerous proteins, resulting in their diverse functions (9). An important function of DNMs is their ability to activate the production of nitric oxide synthases (NOS) by protein-protein interaction (10). Cao et al (11) demonstrated that the proline-rich carboxylic acid terminal domain of DNM2 can interact with the flavin adenine dinucleotide-binding region of endothelial (e) NOS, activating eNOS and promoting nitric oxide (NO) production. Hyndman et al (10) revealed that DNM1, DNM2 and DNM3 increased the production of $\mathrm{NO}$ by their interaction with neuronal NOS (nNOS). Conversely, Kang-Decker et al (12) identified that NO could also directly promote DNM function by cysteine residue nitrosylation.

The role of DNM3 in malignant tumors is largely unknown. Shen et al (13) demonstrated in 2012 that the promoter of DNM3 was hypermethylated in HCC tissues. To the best of our knowledge, the association between DNM3 and HCC was first described by Inokawa et al (14) in 2013 following a triple combination array analysis. The authors revealed that DNM3 was a candidate tumor suppressor gene against HCC. Hypermethylation of the DNM3 promoter was detected more frequently in HCC tissues compared with in normal tissues, and the relative mRNA expression level of DNM3 in HCC tissues tended to be decreased compared with that in normal tissues. Furthermore, HCC growth was associated with DNM3 expression level, and patients with HCC and decreased DNM3 expression levels had poorer prognosis (14). However, the precise role of DNM3 as a tumor suppressor in HCC has not yet been revealed. 
The present study aimed to evaluate the tumor-suppressive effect of DNM3 on HCC cells and to elucidate the mechanism underlying the DNM3-mediated inhibition of HCC growth.

\section{Materials and methods}

Reagents, cell lines and culture conditions. The primary antibody against DNM3 (cat. no. ab3458) was obtained from Abcam (Cambridge, UK). Primary antibodies against inducible (i)NOS (cat. no. sc-7271), nNOS (cat. no. sc-136006) and $\beta$-actin (cat. no. sc-130300) were purchased from Santa Cruz Biotechnology, Inc. (Dallas, TX, USA). Primary antibodies against caspase-3 (cat. no. 9662), cleaved caspase-3 (cat no. 9664), caspase-9 (cat. no. 9508), cleaved caspase-9 (cat. no. 7237), poly ADP-ribose polymerase (PARP; cat. no. 9532), cleaved PARP (cat. no. 5625), cyclin D1 (cat. no. 2978), cyclin-dependent kinase (CDK)2 (cat. no. 2546), CDK4 (cat. no. 12,790) and GAPDH (cat. no. 5174) were obtained from Cell Signaling Technology, Inc. (Danvers, MA, USA). The iNOS inhibitor L-canavanine was supplied by Sigma-Aldrich (Merck KGaA, Darmstadt, Germany). L-canavanine $(1 \mathrm{mM})$ was incubated with the indicated cells at $37^{\circ} \mathrm{C}$ for $24 \mathrm{~h}$. The L02 human hepatic cell line and HepG2, Hep3B, SMMC-7721, PLC/PRF/5, Bel-7402 and Huh7 human HCC cell lines were obtained from the Cell Bank of the Type Culture Collection of the Chinese Academy of Sciences (Shanghai, China). The cells were cultured in Dulbecco's modified Eagle's medium (Gibco; Thermo Fisher Scientific, Inc., Waltham, MA, USA) supplemented with $10 \%$ fetal bovine serum and maintained in $5 \% \mathrm{CO}_{2} / 95 \%$ air at $37^{\circ} \mathrm{C}$.

Patient tissue samples. Between January 2014 and August 2014, 10 patients with HCC from the Department of General Surgery of Jinshan Hospital (Fudan University, Shanghai, China) who underwent liver resection were recruited to the present study. The patients recruited included 7 male patients and 3 female patients aged 59-72 years (mean age, 67.6 years). All patients were diagnosed with primary $\mathrm{HCC}$ with pathological confirmation. Tumor tissues and the adjacent non-tumor tissues from the patients obtained during resection were used for further research. Written informed consent was obtained from all patients prior to enrollment in the present study. The study was approved by the Medical Ethics Committee of Fudan University.

Animals. In total, 30 male nude mice (age, 4 weeks; weight, 15 to $18 \mathrm{~g}$ ) were purchased from the Shanghai Institute of Material Medicine (Shanghai, China) and housed in pathogen-free conditions with free access to food and water. The animal procedures were performed in accordance with the guidelines established by the Shanghai Medical Experimental Animal Care Commission. The nude mice were divided into 3 groups of 10 . For the generation of subcutaneous tumors, 5x10 ${ }^{6}$ SMMC-7721 cells suspended in $100 \mu \mathrm{l}$ PBS were injected subcutaneously into the upper left flank region of the nude mice. The tumor diameter was determined every 7 days. The tumor volume (V) was calculated by the following formula: $\mathrm{V}=1 / 2 \mathrm{x}$ (largest diameter) $\mathrm{x}$ (smallest diameter) ${ }^{2}$.

Total RNA extraction and reverse transcription-quantitative polymerase chain reaction $(R T-q P C R)$. Total RNA was extracted from patient tissues and cell lines using TRIzol ${ }^{\circledR}$ reagent (Takara Bio, Inc., Otsu, Japan) and reverse transcribed into cDNA using PrimeScript ${ }^{\mathrm{TM}}$ RT Master Mix (Takara Bio, Inc.). The reverse transcription was performed with $5 \mathrm{X}$ PrimeScript RT Master Mix ( $4 \mu \mathrm{l})$, RNA $(1 \mu \mathrm{g})$ and RNase-free $\mathrm{ddH}_{2} \mathrm{O}$, to make $20 \mu \mathrm{l}$ in total. The thermocycler conditions were as follows: $37^{\circ} \mathrm{C}$ for $30 \mathrm{~min}, 85^{\circ} \mathrm{C}$ for $4 \mathrm{sec}$, and $4^{\circ} \mathrm{C}$ for 5 min. Relative mRNA expression level was determined by qPCR with a SYBR ${ }^{\circledR}$ Premix Ex Taq ${ }^{\mathrm{TM}}$ Tli RNaseH Plus PCR kit (Takara Bio, Inc.) and an Applied Biosystems Prism ${ }^{\circledR} 7300$ Sequence Detector (Thermo Fisher Scientific, Inc.). qPCR was performed in triplicate for each sample in a $20 \mu \mathrm{l}$ reaction system which consisted of template cDNA $(2 \mu \mathrm{l})$, primers (1 $\mu \mathrm{M})$, SYBR Premix Ex Taq $(10 \mu \mathrm{l})$, ROX Reference Dye $(0.4 \mu \mathrm{l})$ and $\mathrm{ddH}_{2} \mathrm{O}(7.6 \mu \mathrm{l})$. The thermocycler settings were as follows: an initial cycle of $60 \mathrm{sec}$ at $95^{\circ} \mathrm{C}$, then 45 cycles of $15 \mathrm{sec}$ at $95^{\circ} \mathrm{C}, 15 \mathrm{sec}$ at $58^{\circ} \mathrm{C}$ and $30 \mathrm{sec}$ at $72^{\circ} \mathrm{C}$. GAPDH was used as the internal control. The relative mRNA level was determined by the $2^{-\Delta \Delta \mathrm{Cq}}$ method (15). The sequences $\left(5^{\prime}-3^{\prime}\right)$ of the primers were as follows: DNM3 forward, TCGAGGGTCGGG CATTGTA; DNM3 reverse, CTTCAATCTCAAGGCGAAC TTCA; GAPDH forward, TGTGGGCATCAATGGATTTGG; and $G A P D H$ reverse, ACACCATGTATTCCGGGTCAAT.

Western blotting. Total protein was extracted using NP40 solution (cat. no. P0013F; Beyotime Institute of Biotechnology, Haimen, China). Western blotting was performed as described in an existing protocol (16). Following transfer to polyvinylidine fluoride (PVDF) membranes, the protein levels were determined by incubation with the previously described primary antibodies overnight at $4^{\circ} \mathrm{C}$. All primary antibodies were used at a dilution of 1:500, with the exception of GADPH, which was used at a dilution of 1:1,000. Subsequent to washing with TBS containing Tween 20 (TBST), the PVDF membranes were incubated with the corresponding horseradish peroxidase-conjugated secondary antibodies (dilution, 1:10,000) for $2 \mathrm{~h}$ at room temperature, including HRP-linked anti-rabbit IgG (cat. no. 7074) or HRP-linked anti-mouse IgG (cat. no. 7076) from Cell Signaling Technology, Inc. (Danvers, MA, USA). Subsequent to washing with TBST, the signals were developed using an enhanced chemiluminescence system (ZSGB-Bio; OriGene China, Beijing, China) and visualized using the Tanon 5200 Chemiluminescent Imaging System (Tanon Science and Technology Co., Ltd., Shanghai, China).

Cell viability assay. SMMC-7721 cells were seeded on 96-well plates at a density of $5 \times 10^{3}$ cells/well and incubated with Cell Counting Kit-8 (CCK-8) reagent (Dojindo Molecular Technologies, Inc., Kumamoto, Japan) at $37{ }^{\circ} \mathrm{C}$ for $2 \mathrm{~h}$. The absorbance values at $490 \mathrm{~nm}$ were evaluated by using a microplate reader (Omega Bio-tek, Inc., Norcross, GA, USA). All experiments were performed in triplicate.

Cell cycle analysis. Cell cycle analysis was performed using a cell cycle kit (cat. no. C1052; Beyotime Institute of Biotechnology, Haimen, China). SMMC-7721 cells were washed with cold PBS three times and then fixed in ice-cold $70 \%$ ethanol at $-20^{\circ} \mathrm{C}$ for $12 \mathrm{~h}$. Following fixation, cells were resuspended in cold PBS supplemented with 200 mg/ml ribonuclease (cat. no. C1052-3; Beyotime Institute 
of Biotechnology) and stained with $0.5 \mathrm{ml}$ propidium iodide (PI) staining buffer at $37^{\circ} \mathrm{C}$ for $30 \mathrm{~min}$ in the dark. Cell cycle analysis was determined using a BD FACSVerse flow cytometer (BD Biosciences, Franklin Lakes, NJ, USA). The populations of cells distributed in the $G_{0} / G_{1}, S$ and $G_{2} / M$ cell cycle phases were evaluated by FlowJo software (version 7.6.1; Tree Star, Inc., Ashland, OR, USA). All experiments were repeated three times.

Annexin V-PI assay. The Annexin V-PI assay was performed using a commercial Annexin V-FITC Apoptosis Detection kit (cat. no. C1062; Beyotime Institute of Biotechnology). SMMC-7721 cells were resuspended in cold PBS and harvested by centrifugation at $157 \mathrm{x}$ g for $5 \mathrm{~min}$ at $4^{\circ} \mathrm{C}$. The cells were then washed with PBS and resuspended in $300 \mu$ l binding buffer from the kit. Subsequently, the cells were incubated with $5 \mu \mathrm{l}$ Annexin V-FITC and $5 \mu \mathrm{l}$ PI in the dark for $15 \mathrm{~min}$ and subjected to flow cytometry.

Short hairpin (sh)RNA transfection. Human DNM3 shRNA and a scrambled shRNA control were purchased from Santa Cruz Biotechnology, Inc. and transfected into SMMC-7721 cells using Lipofectamine 3000 (Thermo Fisher Scientific, Inc.), according to the manufacturer's protocol.

DNM3 overexpression. The pLenti-DNM3 cDNA lentiviral vector was purchased from Shanghai GenePharma Co., Ltd. (Shanghai, China). The vector included an enhanced green fluorescent protein (EGFP) tag. The pLenti-DNM3 cDNA vector and negative control (NC) vector (EGFP only) were transfected into SMMC-7721 cells, respectively.

Determination of NO production. NO production was detected using a commercial NO detection kit (cat. no. S0021; Beyotime Institute of Biotechnology, Haimen, China) to evaluate nitrite accumulation in the culture medium supernatant, using Griess reagent following conversion of nitrate into nitrite by nitrate reductase. Absorbance values at $540 \mathrm{~nm}$ were evaluated using a microplate reader (Omega Bio-tek, Inc., Norcross, GA, USA).

Evaluation of ROS generation. Intracellular expression levels of ROS were determined by the oxidative conversion of 2',7'-dichlorofluorescein diacetate (DCFH-DA) to the highly fluorescent compound 2',7'-dichlorofluorescein (DCF). Following their harvesting and washing in PBS, the cells were incubated with $10 \mu \mathrm{M}$ DCFH-DA for $20 \mathrm{~min}$ at $37^{\circ} \mathrm{C}$ in the dark. DCF fluorescence was evaluated using a GLOMAX Multidetection System (Promega Corporation, Madison, WI, USA) with a blue filter (excitation $490 \mathrm{~nm}$; emission 510-570 nm).

Mitochondrial membrane potential assay. The mitochondrial membrane potential was detected with a commercial 5,5',6,6'-tetrachloro-1,1',3,3'-tetraethyl-imidacarbocyanine iodide (JC-1) probe kit used according to the manufacturer's protocol (cat. no. C2006; Beyotime Institute of Biotechnology). Briefly, cells seeded in 96-well plates were incubated with JC-1 $(5 \mu \mathrm{g} / \mathrm{ml})$ at $37^{\circ} \mathrm{C}$ for $20 \mathrm{~min}$. The mitochondrial membrane potential was determined by the relative intensity of dual emissions from mitochondrial JC-1 monomers or aggregates under a fluorescence microscope with a $488 \mathrm{~nm}$ excitation wavelength.

Intracellular $\mathrm{Ca}^{2+}$ concentration detection. Intracellular $\mathrm{Ca}^{2+}$ concentration was determined with Fluo-3/acetoxymethyl ester (Fluo-3/AM; cat. no. S1056; Beyotime Institute of Biotechnology) staining conducted as according to the manufacturer's protocol. Briefly, cells were suspended in Tyrode's buffer and incubated with $5 \mu \mathrm{M}$ Fluo-3/AM at room temperature for $30 \mathrm{~min}$, in the dark. The extent of Fluo-3 fluorescence in cells was then analyzed with a FACSCalibur flow cytometer (BD Biosciences).

Immunohistochemistry (IHC). The iNOS protein levels in the subcutaneous tumors of nude mice were determined by IHC. The nude mice were sacrificed 42 days after the generation of the xenograft model, and the tumors were excised, fixed in $4 \%$ paraformaldehyde and embedded in paraffin. Subsequently, tumor tissue sections of $5-\mu \mathrm{m}$ thickness were obtained. IHC was performed according to a previously published protocol (17). A primary antibody against iNOS was used at a dilution of 1:200. Sections were incubated with the primary antibody at $4^{\circ} \mathrm{C}$ overnight. After washing with TBST, the sections were incubated with the aforementioned HRP-linked anti-mouse $\mathrm{IgG}$ at room temperature for $2 \mathrm{~h}$ (dilution, 1:10,000). Reactivity was visualized with 3,3'-diaminobenzidine staining at room temperature for $10 \mathrm{~min}$. The sections were counterstained with hematoxylin. Pathological changes were photographed with a BX51 light microscope (Olympus Corporation, Tokyo, Japan).

Statistical analysis. The data are expressed as the means of 3 repetitions \pm standard deviation, and differences between groups were analyzed using a Student's t-test or a one-way analysis of variance. $\mathrm{P}<0.05$ was considered to indicate a statistically significant difference.

\section{Results}

DNM3 is downregulated in human HCC tissues and cells. In order to investigate the role of DNM3 in HCC proliferation, the present study first evaluated DNM3 expression levels in 10 pairs of HCC tissues and the peritumoral tissues. RT-qPCR demonstrated a downregulation of DNM3 mRNA in the tumor tissues compared with that in the adjacent tissues $(\mathrm{P}=0.0384$; Fig. 1A). In addition, decreased DNM3 protein expression levels in were detected in HCC tumor tissues compared with their paired tumor-adjacent tissues (Fig. 1B).

Subsequently, DNM3 expression levels in 6 human $\mathrm{HCC}$ cell lines were evaluated. The DNM3 mRNA expression levels in the L02 human hepatic cell line was used as the control. It was revealed that DNM3 expression levels were significantly decreased in HepG2, Hep3B, SMMC-7721, Bel-7402 and Huh7 cells compared with L02 cells $(\mathrm{P}<0.05$; Fig. 1C); however, DNM3 was not significantly decreased in $\mathrm{PLC} / \mathrm{PRF} / 5$ cells. The protein expression levels of DNM3 were also decreased in HCC cell lines compared with L02 cells (Fig. 1D). These results were consistent with the findings by Inokawa et al (14) that DNM3 was downregulated in human HCC tissues. 

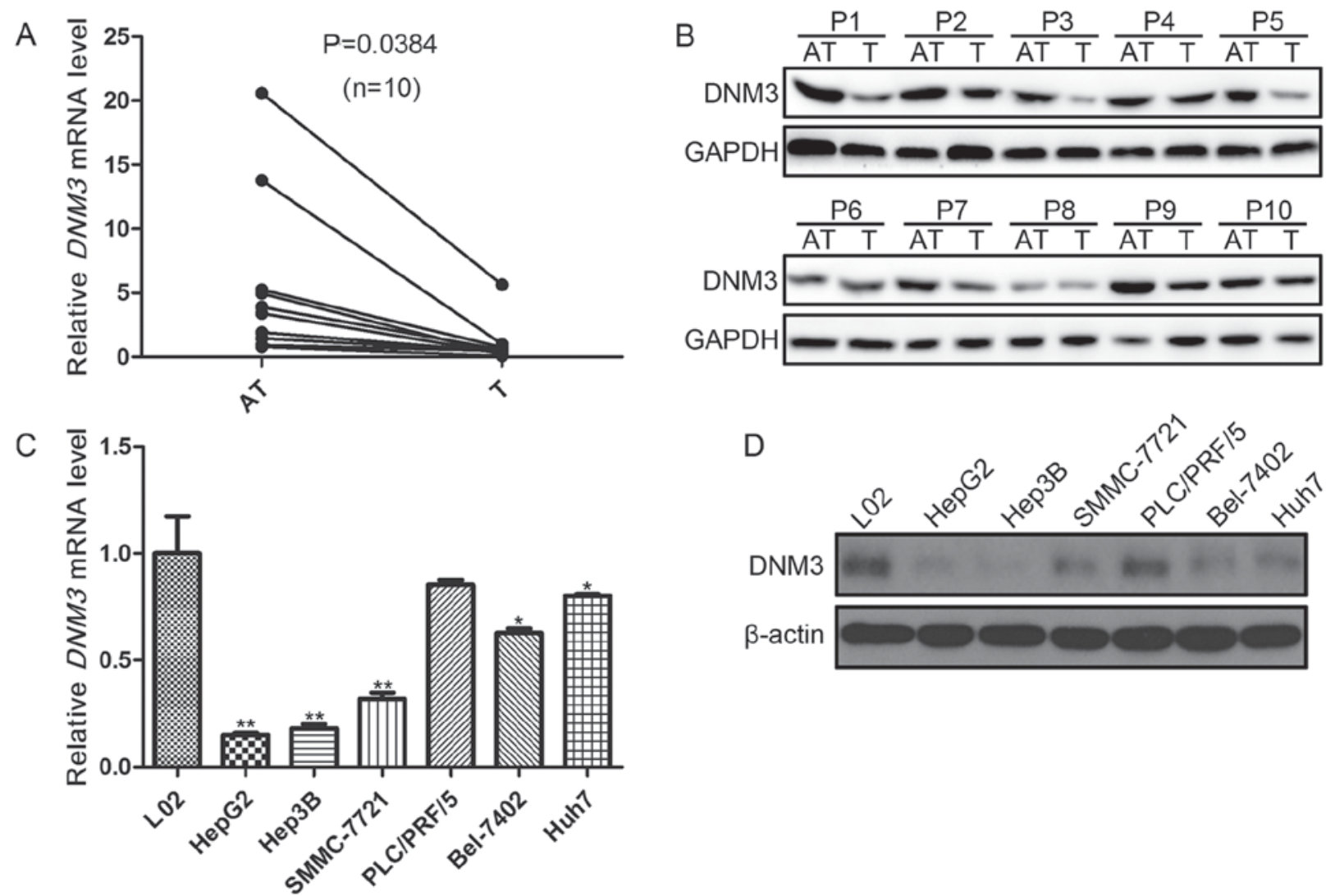

Figure 1. DNM3 expression levels in human HCC tissue samples and cells. (A) Relative DNM3 mRNA expression levels in 10 pairs of HCC tumor and tumor-adjacent tissues, determined by RT-qPCR. (B) Western blotting results of DNM3 expression in 10 pairs (P1-10) of HCC tumor and tumor-adjacent tissues. (C) DNM3 mRNA expression profiles in six human HCC cell lines and L02 human liver cells (set as the control), determined by RT-qPCR ("P $<0.05$ and ${ }^{* *} \mathrm{P}<0.01$ vs. L02 cells). (D) Western blotting results of DNM3 expression in six human HCC cell lines and L02 cells. DNM3, dynamin 3; HCC, hepatocellular carcinoma; T, tumor tissue; AT, tumor-adjacent tissue; RT-qPCR, reverse transcription-quantitative polymerase chain reaction.

Inhibition of DNM3 in SMMC-7721 cells promotes cell proliferation. In order to investigate the effects of DNM3 downregulation on $\mathrm{HCC}$ cells, the present study used DNM3 shRNA to interrupt DNM3 expression in SMMC-7721 cells, as these cells exhibited a moderate DNM3 expression level. The interference efficiency of DNM3 shRNA was confirmed by RT-qPCR and western blotting, which demonstrated that $D N M 3$ shRNA significantly downregulated DNM3 at the mRNA and protein levels (Fig. 2A and B). As previous studies had suggested that DNM3 may serve as a tumor suppressor of HCC, we hypothesized that the downregulation of DNM3 may promote HCC cell proliferation. To verify this hypothesis, a CCK- 8 assay was used to evaluate cell viability. It was revealed that the downregulation of $D N M 3$ increased cell proliferation capacity (Fig. 2C). To investigate the possible mechanism by which the downregulation of DNM3 promoted HCC cell proliferation, the cell cycle distribution of SMMC-7721 cells was analyzed by flow cytometry. The shRNA-mediated downregulation of DNM3 significantly reduced the percentage of SMMC-7721 cells in the $G_{0} / G_{1}$ cell cycle phase, and increased the percentage of cells in the $\mathrm{G}_{2} / \mathrm{M}$ phase (Fig. 2D). Furthermore, the protein expression levels of cell cycle-associated regulatory molecules were determined, demonstrating that a decrease in DNM3 increased the expression levels of cyclin D1, CDK2 and CDK4 compared with the control groups (Fig. 2E). Collectively, these results indicate that the downregulation of DNM3 may promote HCC cell proliferation by increasing cell cycle-associated regulatory proteins.

Overexpression of DNM3 in SMMC-7721 cells induces apoptosis and suppresses tumor growth. To further evaluate the tumor-suppressive effect of DNM3 on HCC cells, DNM3 was overexpressed by introducing the DNM3 cDNA vector into SMMC-7721 cells. At $96 \mathrm{~h}$ after transfection, the overexpression efficiency was determined by RT-qPCR and western blotting. The transfection of the DNM 3 cDNA vector increased DNM3 mRNA and protein expression levels in SMMC-7721 cells compared with the controls (Fig. 3A and B). As expected, the CCK-8 assay revealed that overexpression of DNM3 inhibited SMMC-7721 cell viability (Fig. 3C).

Flow cytometric analysis revealed that the ratio of apoptotic cells was increased in DNM3-overexpressing cells (13.1\%) as compared with NC-transfected cells (1.3\%; Fig. 3D). Subsequently, the protein expression levels of apoptotic proteins were evaluated by western blotting. In SMMC-7721 cells with DNM3 overexpression, the cleaved forms of caspase-3, caspase-9 and PARP increased compared with the control and NC groups (Fig. 3E), indicating that the overexpression of DNM3 activated caspase-dependent apoptosis. The tumor-suppressive effect of DNM3 in HCC was further verified in animal models; DNM3 overexpression significantly inhibited subcutaneous tumor growth relative to the control groups in nude mice (Fig. 3F). 


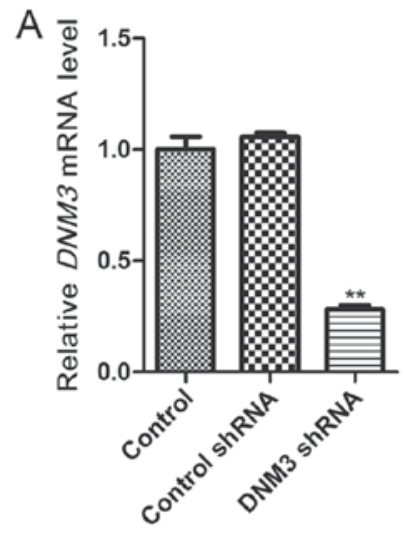

B

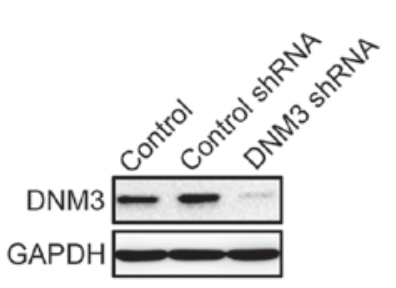

D

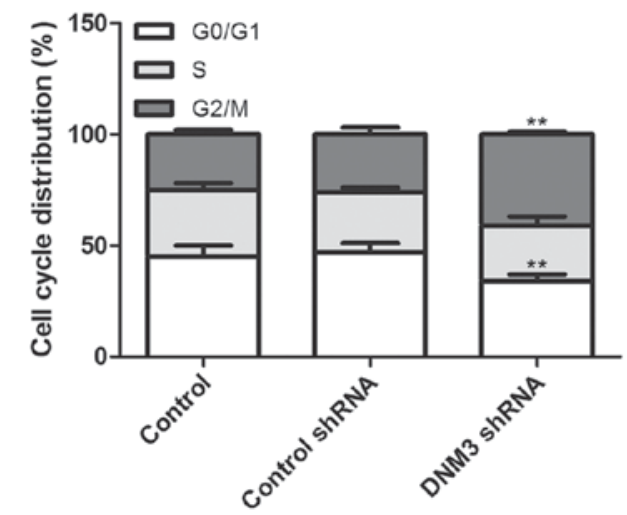

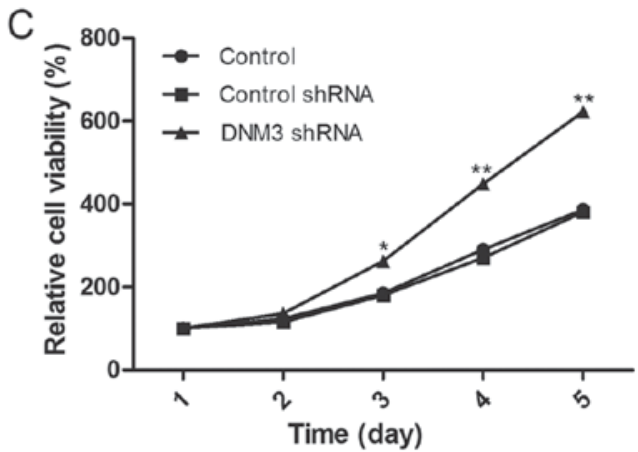

E

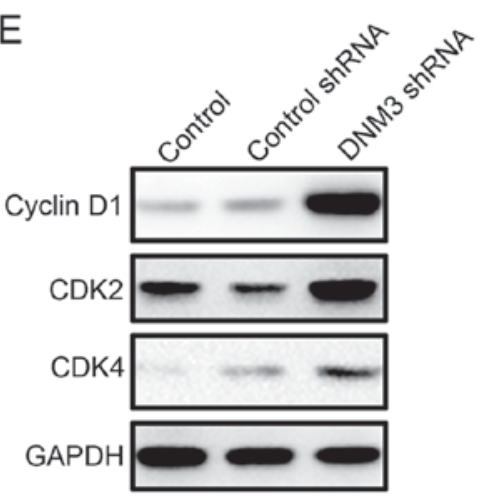

Figure 2. Inhibition of DNM3 promotes SMMC-7721 HCC cell proliferation. (A) DNM3 interference efficiency of DNM3 shRNA confirmed by (A) reverse transcription-quantitative polymerase chain reaction and (B) western blotting. (C) Effect of DNM3 downregulation on SMMC-7721 cell viability. (D) Inhibition

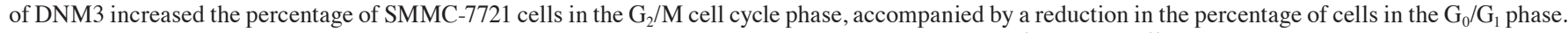
(E) Inhibition of DNM3 in SMMC-7721 cells increased cyclin D1, CDK2 and CDK4 expression levels. ${ }^{*} \mathrm{P}<0.05$ and ${ }^{* *} \mathrm{P}<0.01$ vs. control shRNA group. DNM3, dynamin 3; HCC, hepatocellular carcinoma; shRNA, short hairpin RNA; CDK, cyclin-dependent kinase.

Overexpression of DNM3 induces intracellular ROS accumulation. The aforementioned results revealed that the downregulation of DNM3 in HCC cells promoted cell proliferation, whereas the overexpression of DNM3 induced HCC cell apoptosis and inhibited HCC growth. Subsequently, the present study aimed to elucidate the potential mechanism by which DNM3 upregulation induced HCC cell apoptosis. It has previously been reported that mitochondrial damage is an early event in cellular death and that intracellular ROS accumulation is a biochemical mediator of apoptosis which could trigger cell death (18-20); thus, the present study investigated the mitochondrial membrane potential, cytosolic free $\mathrm{Ca}^{2+}$ concentration and ROS accumulation in SMMC-7721 cells with DNM3 overexpression. The results demonstrated that mitochondrial membrane potential was significantly decreased and free $\mathrm{Ca}^{2+}$ concentration significantly increased following DNM3 overexpression (Fig. 4A and B). Significantly elevated intracellular ROS accumulation in DNM3-overexpressing SMMC-7721 cells was also observed (Fig. 4C). These results indicate that DNM3 overexpression can impair mitochondrial function and promote ROS accumulation.

Overexpression of DNM3 induces iNOS and NO production. It has been previously reported that the accumulation of intracellular ROS increases with NO $(21,22)$, and that DNM3 can activate NO production (10). Therefore, we hypothesized that increased ROS accumulation and apoptosis in DNM3-overexpressing cells may be due to increased NO. Nitrite levels in the culture supernatants of SMMC-7721 cells overexpressing DNM3 were detected. Increased nitrite in the supernatants, indicating elevated $\mathrm{NO}$ production, were detected following DNM3 upregulation in SMMC-7721 cells (Fig. 5A).

Subsequently, the protein expression levels of NOS following DNM3 overexpression were investigated. According to a study by Heymann and Hinshaw (7), DNM3 may interact with nNOS directly and activate NO production. However, in the present study, the protein expression level of nNOS was the same following DNM3 overexpression. The protein expression level of iNOS, by contrast, was markedly increased in DNM3-overexpressing SMMC-7721 cells (Fig. 5B). Furthermore, the expression level of iNOS in subcutaneous tumors generated from DNM3-overexpressing SMMC-7721 cells also increased, as revealed by IHC staining (Fig. 5C). These results demonstrated that increased NO production may induce ROS accumulation, and may result from increased iNOS production.

Inhibition of iNOS attenuates DNM3-induced apoptosis. To investigate whether the increased $\mathrm{NO}$ and ROS expression levels were the results of iNOS activation, the specific iNOS inhibitor L-canavanine was utilized for further experiments. Incubation with L-canavanine significantly attenuated the DNM3-induced mitochondrial membrane potential decrease 
A

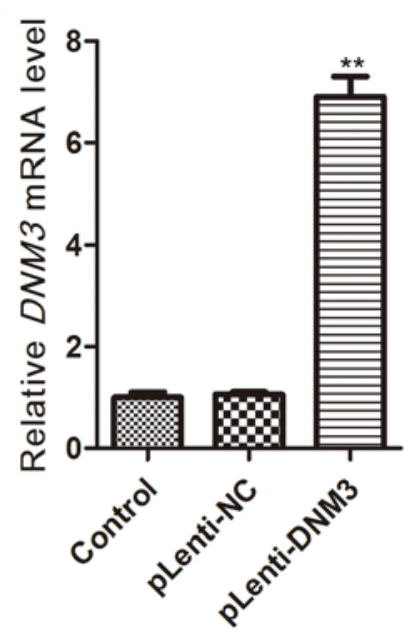

B

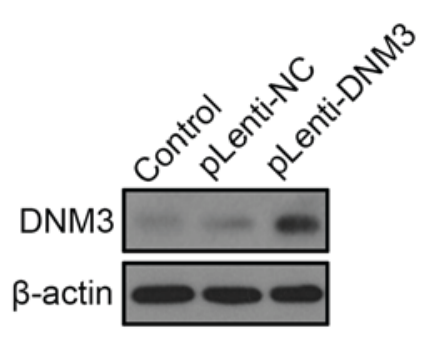

C

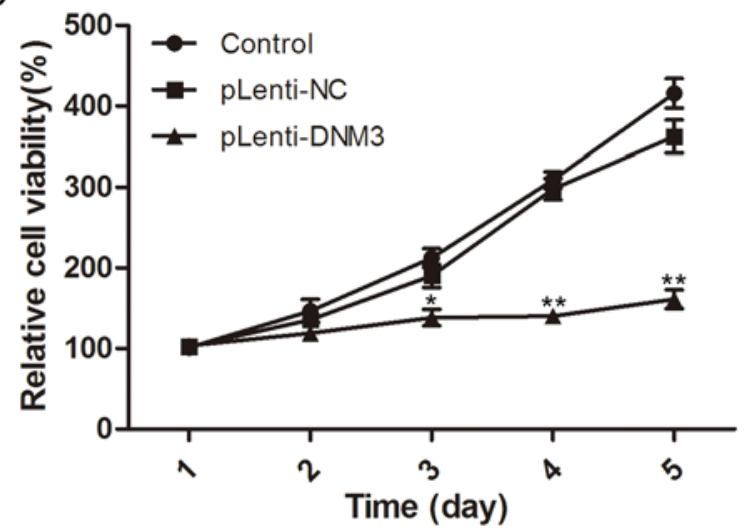

$\mathrm{D}$

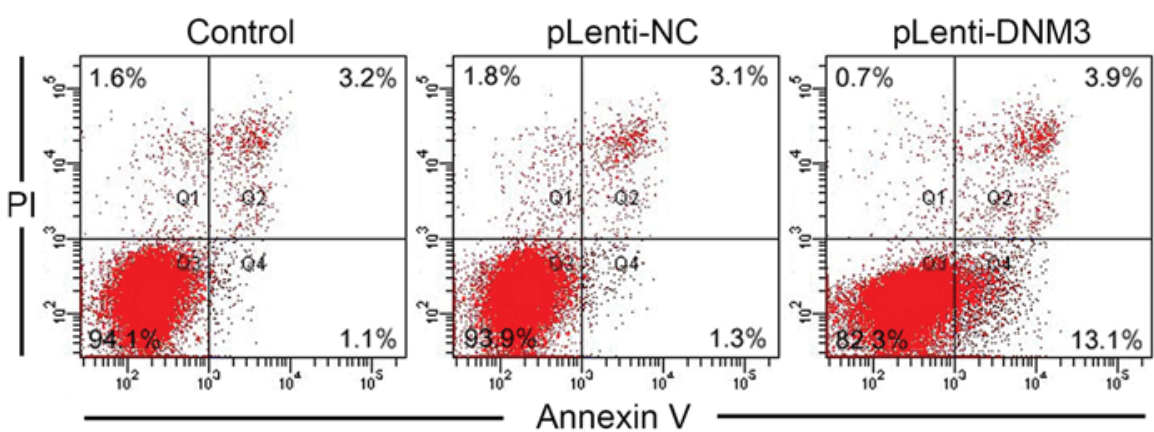

$\mathrm{F}$

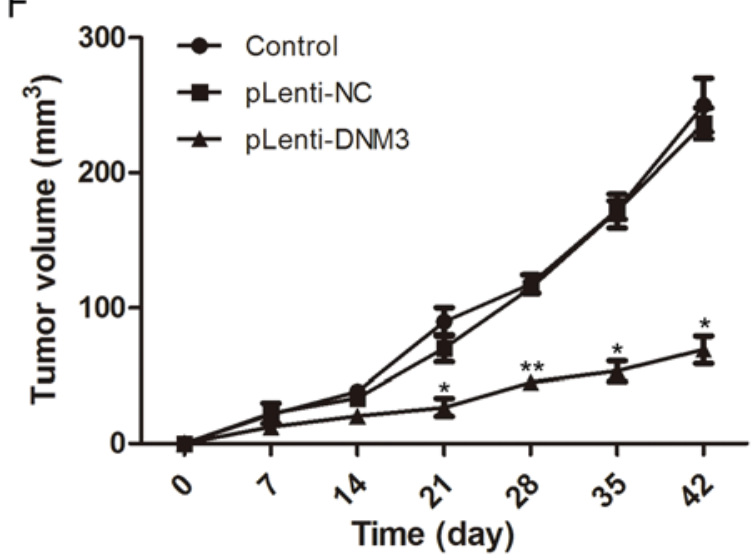

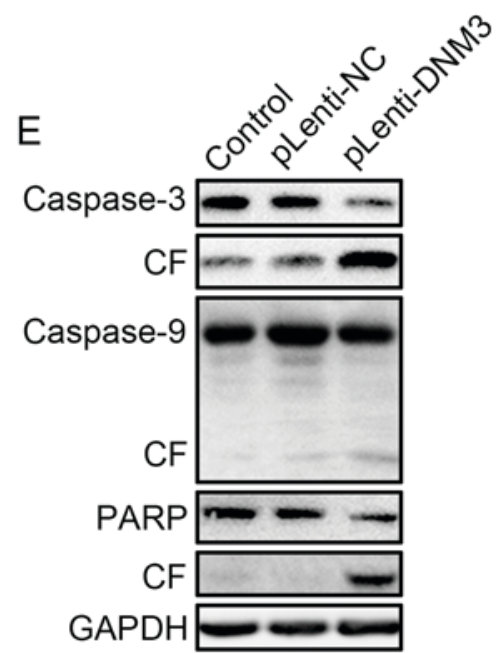



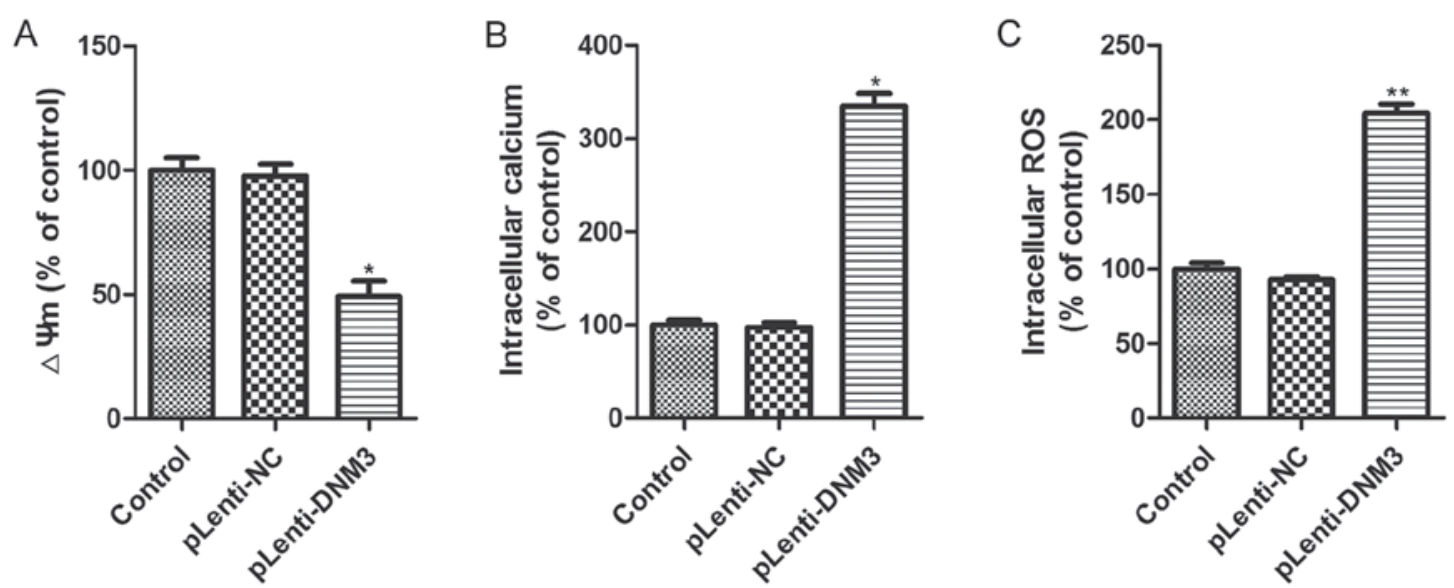

Figure 4. Effect of DNM3 upregulation on $\Delta \Psi \mathrm{m}$, accumulation of intracellular $\mathrm{Ca}^{2+}$ and ROS. (A) $\Delta \Psi \mathrm{m}$ of SMMC-7721 cells with DNM3 overexpression was evaluated by JC-1 red fluorescence. DNM3 overexpression decreased the $\Delta \Psi \mathrm{m}$. (B) Intracellular $\mathrm{Ca}^{2+}$ concentration in DNM3-overexpressing cells was determined using Fluo-3AM fluorescence. DNM3 overexpression increased intracellular $\mathrm{Ca}^{2+}$ concentration. (C) Intracellular ROS concentration was determined using the $2^{\prime}, 7^{\prime}$-dichlorofluorescein diacetate fluorescent probe. DNM3 overexpression increased intracellular ROS levels. ${ }^{*} \mathrm{P}<0.05$ and ${ }^{* *} \mathrm{P}<0.01$ vs. pLenti-NC group. DNM3, dynamin 3; $\Delta \Psi \mathrm{m}$, mitochondrial membrane potential; ROS, reactive oxygen species; pLenti-DNM3, lentivirus containing DNM3; pLenti-NC, negative control lentivirus.

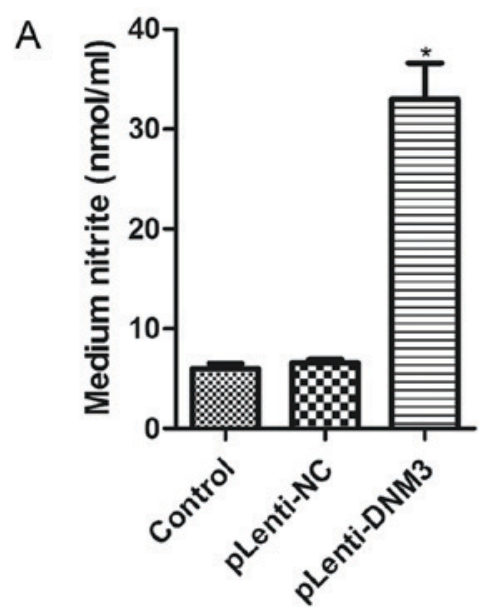

C

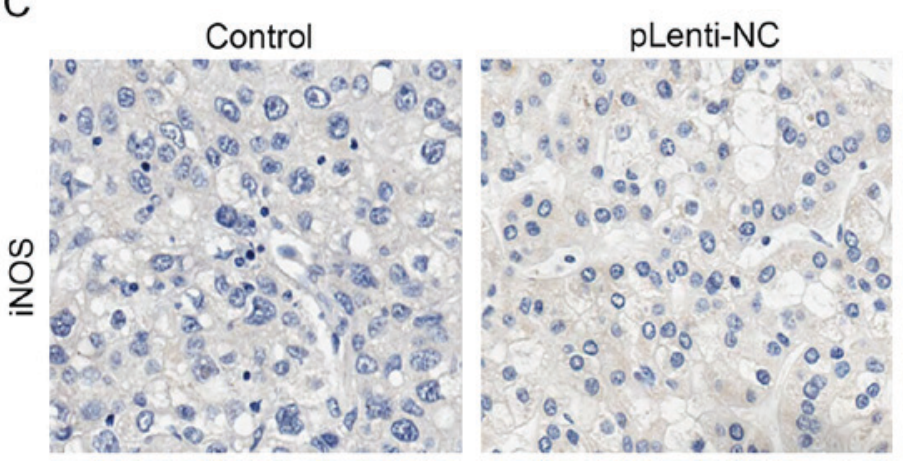

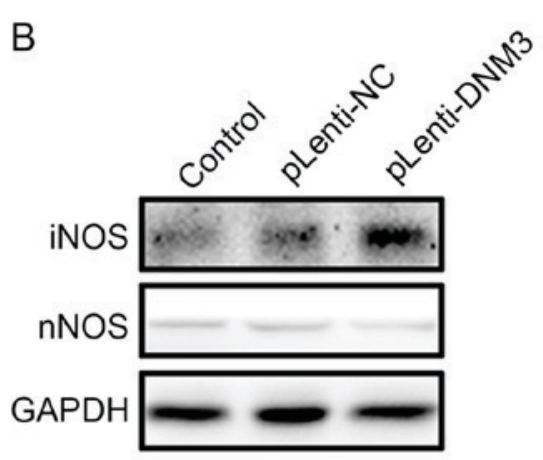

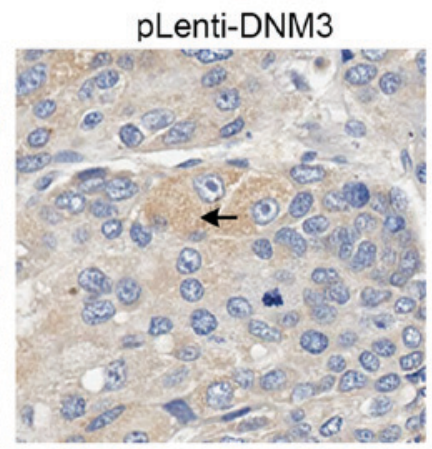

Figure 5. Overexpression of DNM3 in SMMC-7721 cells increased iNOS and NO production. (A) Nitrite levels in the medium supernatants were evaluated using the Griess reaction. Overexpression of DNM3 significantly increased nitrite levels (" $\mathrm{P}<0.05$ vs. pLenti-NC group). (B) Western blotting results for iNOS and nNOS levels in SMMC-7721 cells with DNM3 overexpression. (C) Immunohistochemical staining results of iNOS in nude mouse subcutaneous tumor tissues generated from SMMC-7721 cells with or without DNM3 overexpression. Magnification, $\mathrm{x} 400$. The arrow indicates positive staining for iNOS. DNM3, dynamin 3; iNOS, inducible nitric oxide synthase; NO, nitric oxide; nNOS, neuronal nitric oxide synthase; pLenti-DNM3, lentivirus containing DNM3; pLenti-NC, negative control lentivirus.

\section{Discussion}

The present study demonstrated that DNM3 was downregulated in HCC tissues and cells. The downregulation of
DNM3 promoted cell proliferation by increasing certain cell cycle-associated proteins. By contrast, overexpression of DNM3 inhibited HCC cell viability and induced apoptosis. The in vivo experiments also demonstrated that overexpression of 

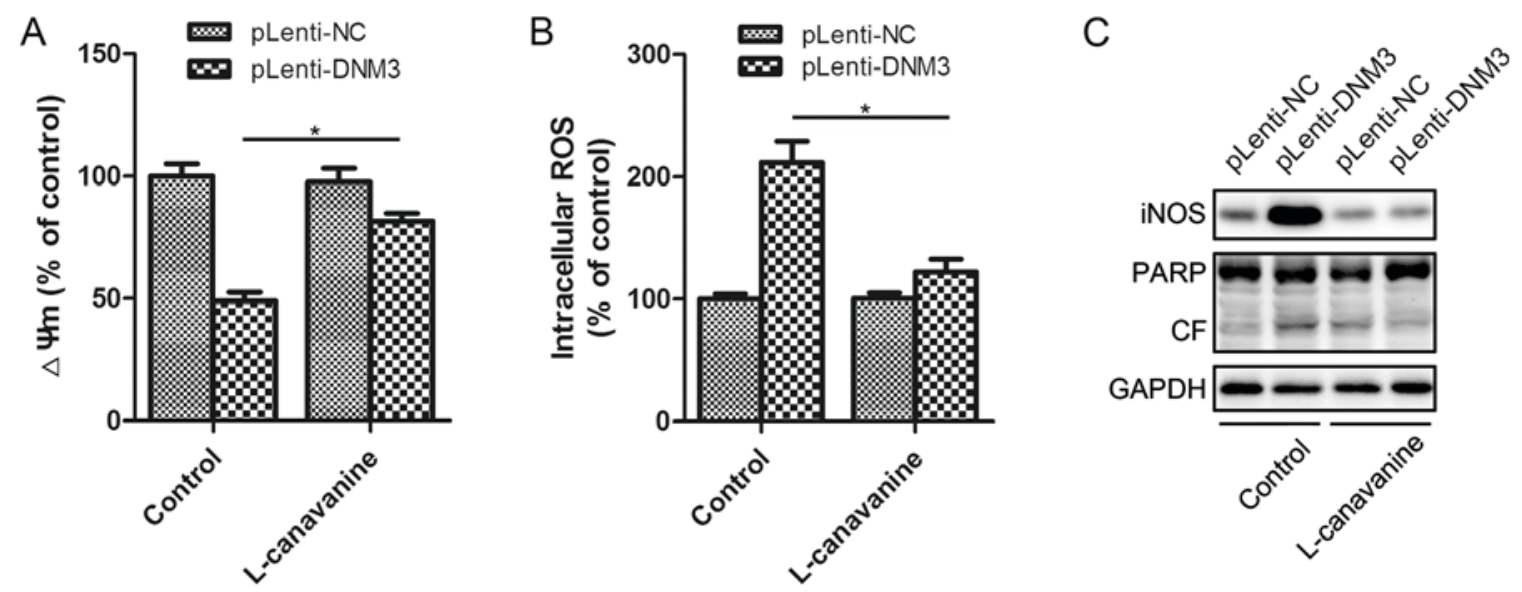

Figure 6. Inhibition of iNOS attenuated DNM3-overexpression-induced apoptosis. (A) The iNOS inhibitor, L-canavanine, attenuated the DNM3-induced decrease in $\triangle \Psi \mathrm{m}$. (B) L-canavanine inhibited the DNM3-induced increase in intracellular ROS levels. (C) L-canavanine inhibited a DNM3-induced increase in cleaved PARP in SMMC-7721 cells. ${ }^{*}<<0.05$. DNM3, dynamin 3; iNOS, inducible nitric oxide synthase; $\Delta \Psi \mathrm{m}$, mitochondrial membrane potential; ROS, reactive oxygen species; pLenti-DNM3, lentivirus containing DNM3; pLenti-NC, negative control lentivirus; PARP, poly ADP-ribose polymerase; $\mathrm{CF}$, cleaved form.

DNM3 suppressed the growth of human HCC xenograft tumors in nude mice. Furthermore, the mechanisms underlying the DNM3-mediated inhibition of HCC growth was investigated. This revealed that increased iNOS expression levels following DNM3 overexpression were associated with decreased mitochondrial membrane potentials and increased accumulation of intracellular ROS. These effects could be attenuated by the iNOS inhibitor L-canavanine. Thus, DNM3 can inhibit HCC by activating iNOS and subsequent NO production, inducing ROS accumulation and HCC cell apoptosis.

$\mathrm{NO}$ is one of the smallest signaling molecules and is involved in various physiological functions (23). As previously reported, NO serves a dual role in tumor biological behavior: Prolonged and excessive NO levels may lead to inflammation and tumor development, whereas higher expression levels of NO could also trigger cell apoptosis $(24,25)$. NO may be generated in NOS-dependent and NOS-independent manners $(23,26)$. The NOS-independent signaling pathway involves the non-enzymatic generation of NO; NO produced from dietary sources of nitrate and nitrite in the bloodstream and tissues complements the NOS-dependent signaling pathway (26). NOSs are enzymes that catalyze the formation of NO from nitrogen in guanidine and L-arginine $(27,28)$. There are three forms of NOS: nNOS, iNOS and eNOS (23). The present study demonstrated that DNM3 increased the level of iNOS but not nNOS, in contrast to a previous study by Hyndman et al which suggested that DNM3 activated nNOS (10). Previous studies have revealed that nNOS and eNOS are constitutive NOS isoforms (cNOS), and may be stimulated by a variety of signaling pathways that are dependent or independent of $\mathrm{Ca}^{2+}(29)$. However, iNOS may be induced by pathological stimuli which are $\mathrm{Ca}^{2+}$-independent in numerous types of cells, including hepatocytes, Kupffer cells, lymphocytes and macrophages (30). One of the major differences between iNOS and cNOS is that iNOS is capable of releasing a large amount of $\mathrm{NO}$ for relatively long periods of time in a sustained manner, whereas cNOS produces a small amount of NOS over a short period of time and is short-acting (31-33). The present study demonstrated that increased expression levels of iNOS after
DNM3 upregulation induced apoptosis, whereas the inhibition of iNOS via L-canavanine attenuated the ROS accumulation and apoptosis. Janakiram and Rao (23) reported that high levels of iNOS expression may exert cytostatic or cytotoxic effects on tumor cells; thus, the increased iNOS expression levels following DNM3 overexpression were likely a crucial step in the induction of SMMC-7721 cell apoptosis. However, the present study did not elucidate the pathway underlying DNM3-activated iNOS production. This may be due to a direct interaction between DNM3 and iNOS (11).

We hypothesized that impaired mitochondrial function and intracellular ROS accumulation may serve a vital role in inducing apoptotic cell death following DNM3 upregulation. Mitochondrial damage is an early event in cellular death (18). The decrease in mitochondrial membrane potential occurs earlier than the morphological alterations of mitochondria and DNA fragmentation. Increased membrane permeability decreases mitochondrial membrane potential, and this is closely associated with the opening of the mitochondrial permeability transition pore (MPTP). Irreversible opening of the MPTP has previously been revealed to be involved in cell apoptosis (34). Mitochondria are regarded as the main source of ROS in tumor cells $(35,36)$. It was previously demonstrated that an increased expression level of intracellular ROS was a biochemical mediator of apoptosis and was sufficient to trigger cell death (37). Excessive ROS accumulation has also been revealed to alter cell functions and disrupt the intracellular homeostasis of the redox system via oxidative damage (38). Cohen and Vanhoutte (28) reported that ROS mediated the early and late steps of apoptosis associated with mitochondrial dysfunction. In the present study, an increased DNM3 expression level was revealed to decrease mitochondrial membrane potential and increase intracellular ROS accumulation.

In conclusion, the present study demonstrated that DNM3 can inhibit the proliferation of SMMC-7721 cells and induce apoptosis by activating iNOS production and, subsequently, an increase in NO expression level and ROS accumulation. Further studies are required in order to reveal the interactions between DNM3 and iNOS. 


\section{Acknowledgements}

The present study was supported by the Health and Family Commission of Shanghai Jinshan District (grant no. JSKJ-KTMS-2014-06).

\section{References}

1. Torre LA, Bray F, Siegel RL, Ferlay J, Lortet-Tieulent J and Jemal A: Global cancer statistics, 2012. CA Cancer J Clin 65: 87-108, 2015

2. Earl TM and Chapman WC: Hepatocellular carcinoma: Resection versus transplantation. Semin Liver Dis 33: 282-292, 2013.

3. Cusnir M and Patt YZ: Novel systemic therapy options for hepatocellular carcinoma. Cancer J 10: 97-103, 2004.

4. Yu MC and Yuan JM: Environmental factors and risk for hepatocellular carcinoma. Gastroenterology 127 (5 Suppl 1): S72-S78, 2004.

5. Shiraha H, Yamamoto K and Namba M: Human hepatocyte carcinogenesis (Review). Int J Oncol 42: 1133-1138, 2013.

6. El-Serag HB and Rudolph KL: Hepatocellular carcinoma: Epidemiology and molecular carcinogenesis Gastroenterology 132: 2557-2576, 2007.

7. Heymann JA and Hinshaw JE: Dynamins at a glance. J Cell Sci 122: 3427-3431, 2009.

8. Hinshaw JE: Dynamin and its role in membrane fission. Annu Rev Cell Dev Biol 16: 483-519, 2000.

9. Schmid SL and Frolov VA: Dynamin: Functional design of a membrane fission catalyst. Annu Rev Cell Dev Biol 27: 79-105, 2011.

10. Hyndman KA, Musall JB, Xue J and Pollock JS: Dynamin activates $\mathrm{NO}$ production in rat renal inner medullary collecting ducts via protein-protein interaction with NOS1. Am J Physiol Renal Physiol 301: F118-F124, 2011.

11. Cao S, Yao J and Shah V: The proline-rich domain of dynamin-2 is responsible for dynamin-dependent in vitro potentiation of endothelial nitric-oxide synthase activity via selective effects on reductase domain function. J Biol Chem 278: 5894-5901, 2003

12. Kang-Decker N, Cao S, Chatterjee S, Yao J, Egan LJ, Semela D, Mukhopadhyay D and Shah V: Nitric oxide promotes endothelial cell survival signaling through S-nitrosylation and activation of dynamin-2. J Cell Sci 120: 492-501, 2007.

13. Shen J, Wang S, Zhang YJ, Kappil M, Wu HC, Kibriya MG, Wang Q, Jasmine F, Ahsan H, Lee PH, et al: Genome-wide DNA methylation profiles in hepatocellular carcinoma. Hepatology 55 1799-1808, 2012

14. Inokawa $Y$, Nomoto S, Hishida M, Hayashi M, Kanda M, Nishikawa Y, Takeda S, Fujiwara M, Koike M, Sugimoto H, et al: Dynamin 3: A new candidate tumor suppressor gene in hepatocellular carcinoma detected by triple combination array analysis. Onco Targets Ther 6: 1417-1424, 2013.

15. Livak KJ and Schmittgen TD: Analysis of relative gene expression data using real-time quantitative PCR and the 2(-Delta Delta C(T)) method. Methods 25: 402-408, 2001.

16. Nie X, Song S, Zhang L, Qiu Z, Shi S, Liu Y, Yao L and Zhu D: 15-Hydroxyeicosatetraenoic acid (15-HETE) protects pulmonary artery smooth muscle cells from apoptosis via inducible nitric oxide synthase (iNOS) pathway. Prostaglandins Other Lipid Mediat 97: 50-59, 2012

17. Yin XY, Jiang JM, Liu JY and Zhu JR: Effects of endogenous nitric oxide induced by 5-fluorouracil and L-Arg on liver carcinoma in nude mice. World J Gastroenterol 13: 6249-6253, 2007.
18. Lemasters JJ: V. Necrapoptosis and the mitochondrial permeability transition: Shared pathways to necrosis and apoptosis. Am J Physiol 276: G1-G6, 1999.

19. Pastorino JG and Hoek JB: Ethanol potentiates tumor necrosis factor-alpha cytotoxicity in hepatoma cells and primary rat hepatocytes by promoting induction of the mitochondrial permeability transition. Hepatology 31: 1141-1152, 2000.

20. Circu ML and Aw TY: Reactive oxygen species, cellular redox systems, and apoptosis. Free Radic Biol Med 48: 749-762, 2010.

21. Lala PK and Chakraborty C: Role of nitric oxide in carcinogenesis and tumour progression. Lancet Oncol 2: 149-156, 2001.

22. Soliman MK, Mazzio E and Soliman KF: Levodopa modulating effects of inducible nitric oxide synthase and reactive oxygen species in glioma cells. Life Sci 72: 185-198, 2002.

23. Vannini F, Kashfi K and Nath N: The dual role of iNOS in cancer. Redox Biol 6: 334-343, 2015.

24. Bonavida B and Baritaki S: Dual role of NO donors in the reversal of tumor cell resistance and EMT: Downregulation of the NF- $\kappa$ B/Snail/YY1/RKIP circuitry. Nitric Oxide 24: 1-7, 2011.

25. Kim SF, Huri DA and Snyder SH: Inducible nitric oxide synthase binds, S-nitrosylates, and activates cyclooxygenase-2. Science 310: 1966-1970, 2005.

26. Lepore DA: Nitric oxide synthase-independent generation of nitric oxide in muscle ischemia-reperfusion injury. Nitric Oxide 4: 541-545, 2000

27. Moncada S, Palmer RM and Higgs EA: Nitric oxide: Physiology, pathophysiology, and pharmacology. Pharmacol Rev 43: 109-142, 1991.

28. Cohen RA and Vanhoutte PM: Endothelium-dependent hyperpolarization. Beyond nitric oxide and cyclic GMP. Circulation 92: 3337-3349, 1995

29. Panda SP, Gao YT, Roman LJ, Martásek P, Salerno JC and Masters BS: The role of a conserved serine residue within hydrogen bonding distance of FAD in redox properties and the modulation of catalysis by $\mathrm{Ca}^{2+} /$ calmodulin of constitutive nitric-oxide synthases. J Biol Chem 281: 34246-34257, 2006.

30. Bredt DS, Hwang PM, Glatt CE, Lowenstein C, Reed RR and Snyder SH: Cloned and expressed nitric oxide synthase structurally resembles cytochrome P-450 reductase. Nature 351: 714-718, 1991.

31. Dominiczak AF and Bohr DF: Nitric oxide and its putative role in hypertension. Hypertension 25: 1202-1211, 1995.

32. Crane BR, Arvai AS, Gachhui R, Wu C, Ghosh DK, Getzoff ED, Stuehr DJ and Tainer JA: The structure of nitric oxide synthase oxygenase domain and inhibitor complexes. Science 278 : 425-431, 1997.

33. Schulz R and Triggle CR: Role of NO in vascular smooth muscle and cardiac muscle function. Trends Pharmacol Sci 15: 255-259, 1994.

34. Crompton M: The mitochondrial permeability transition pore and its role in cell death. Biochem J 341: 233-249, 1999.

35. Higuchi Y: Chromosomal DNA fragmentation in apoptosis and necrosis induced by oxidative stress. Biochem Pharmacol 66: 1527-1535, 2003

36. Bartosz G: Reactive oxygen species: Destroyers or messengers? Biochem Pharmacol 77: 1303-1315, 2009.

37. Buttke TM and Sandstrom PA: Oxidative stress as a mediator of apoptosis. Immunol Today 15: 7-10, 1994.

38. Sabharwal SS and Schumacker PT: Mitochondrial ROS in cancer: Initiators, amplifiers or an Achilles' heel? Nat Rev Cancer 14: 709-721, 2014. 